\title{
Cardiometabolic Diseases: A Global Perspective
}

\author{
Gundu HR Rao* \\ Laboratory Medicine and Pathology, University of Minnesota, USA
}

Submission: June 07, 2018; Published: September 25, 2018

*Corresponding author: Gundu HR Rao, Laboratory Medicine and Pathology, Director of Thrombosis Research, Lillehei Heart Institute, Founder, CEO, South Asian Society on Atherosclerosis and Thrombosis (SASAT), 12500 Park Potomac Ave Unit 306N, Potomac MD 20854, University of Minnesota, USA, Email: Gundurao9@gmail.com

\begin{abstract}
The George Institute for Global Health of Australia, in their website claim that the epidemic of cardio-metabolic diseases is escalating worldwide, including India. The rapid socioeconomic transition is believed to have contributed to this rise, with the individuals being increasingly exposed to energy-dense foods, high stress levels, and sedentary work habits. Larry Husten in an article in Lancet wrote, that in 1960, the typical heart-attack- "victim" was a middle-class US or European executive. Cardiovascular Disease (CVD) was largely unknown in the developing world, but now, a global epidemic of CVD is underway. In the 21st century, say epidemiologists, a typical patient presenting with heart attack will be a Moscow Taxi driver, or a Bombay factory worker [1]. Underlying causes may or may not be the same at each demography. For instance, another article written in the same year, drew the attention of this prediction. Larry Husten contemplated on the enormous rise in deaths from heart disease in Russia. The author of this article points out to the fact, that to a large extent, the pattern of alcohol consumption may play a big role in the cardiovascular deaths in Russia [2]. Since 1980, incidence of obesity has increased by two-fold and diabetes by four-fold worldwide, according to the NCD Risk Factor Collaborators report [3,4]. Hypertension is one of the primary risk factors for heart disease and stroke. Over 875 million were hypertensives in the year 2000 and will double by the year 2025 [5]. Another factor to be considered when discussing from a global perspective, is that nearly two-thirds of individuals with CMDs live in low- and middle-income countries. On the other hand, immigrant population is increasing rapidly in several countries, because of the economic attractiveness and public infrastructure. Just look at the UAE as an example, they have more immigrants than the native Arabs. Although conventional cardiovascular risk factors such as smoking, blood pressure and total cholesterol predict risk within these ethnic groups, they do not fully account for the differences in risk, between ethnic groups, suggesting that alternative explanations might exist. We feel strongly, that there is a great need to investigate the prevalence and pattern of metabolic risks in general, identify the unique risk factors responsible for development and/or progression of these condition's and the economic and social costs for the treatment of these diseases. All of these metabolic diseases have risen to the status of an epidemic, and cost of combating these diseases will pose, in the very near future, a great economic burden to the global community.
\end{abstract}

Keywords: Cardiometabolic Diseases; Cardiovascular Disease; Global epidemic; Epidemiologists; Heart attack; Cardiovascular risk factors; Blood pressure

Abbreviations: CVD: Cardiovascular Disease; AHA: American Heart Association; ASCVD: Atherosclerotic Cardiovascular Disease; UNDP: United Nations Development Program; SASAT: South Asian Society on Atherosclerosis and Thrombosis; IUA: International Union of Angiology; CAD: Coronary Artery Disease; DM: Diabetes Mellitus; ACC: American College of Cardiology; CRF's: Cardiac Risk Factors; NHANES: National Health and Nutrition Examination Survey

\section{Introduction}

Any disturbance in the normal metabolism, leads to alterations in physiology, and manifests in some form of risk factor development in the sequalae of the pathogenesis of metabolic diseases. For instance, if we are discussing altered metabolism, then inflammation, oxidative stress, endothelial dysfunction, hardening of the arteries, calcification of the vessels as well as pericardium, insulin resistance, elevated blood glucose, subclinical atherosclerosis, excess weight, increased visceral fat, increased subcutaneous fat, altered blood lipids, changes in the microbiome, gene expressions favoring development of metabolic risk factors, altered metabolic and signaling pathways at cellular and molecular levels, can be described as metabolic risk factors. However, if we use the term, cardiometabolic risks, then we limit the area of discussion, to the known modifiable risks for developing vascular diseases. By and large, management of vascular diseases, as well as cardiometabolic diseases, to a great extent are limited to reducing, reversing, or prevention of the modifiable risks; such as smoking, hypertension, excess weight, obesity, insulin resistance, hyperglycemia, lipid abnormalities, prediabetes, diabetes, heart disease and stroke [1-11]. All of the metabolic diseases, including excess weight, obesity, metabolic syndrome, type-2 diabetes, and vascular diseases, have achieved epidemic status worldwide 
[1-6]. In a recent Scientific Statement of the American Heart Association, the Chairs, Vice Chair and the committee members of various councils, have put together a comprehensive review on the excess prevalence of type- 2 diabetes, and cardiovascular disease, in South Asians living in the USA [6]. In my opinion, it is high time that, the clinicians, healthcare providers, and policy makers pay attention to the relationship between the various ethnicity and incidence or prevalence of excess metabolic risk, when planning preventive strategies. We have used the excess prevalence of diabetes and CVDs in South Asians immigrants in the US, as an example in this article, to discuss the fundamental question regarding the homogeneity or otherwise of the risks, and the development of CRFs. We applaud the efforts of the Toronto Global Health Research Group of the University of Toronto, and the Department of Health Research Methods, McMaster University, Hamilton ON, Canada, for organizing the first Canadian Ethnicity and Cardiometabolic Disease Conference in Toronto, Canada, in January of this year. We would like to see such conferences organized in the UK, the USA and other countries, where there is considerable increase in various high-risk ethnic populations. In this mini review, we have made an attempt to review some of the salient findings on cardiometabolic diseases in general and presented a global perspective from our point of view [6-11].

In a recent issue of Circulation, a scientific statement from the American Heart Association (AHA) has been put together by Prof. Volgman and associates, on behalf of the AHA, representing Councils on Epidemiology and prevention, Stroke in Women and Special Population, Clinical Cardiology, and various other subspecialties related, to Cardiovascular diseases (CVDs). The title of the article is, "Atherosclerotic Cardiovascular Disease (ASCVD) in South Asians in the United States: Epidemiology, Risk Factors, and Treatments" [6]. Since I have been working on this very topic for over three decades, I decided to start this "mini overview" by commenting on this topic first, and then adding my own point of view. I was invited by the Government of India, in 1990 as a senior consultant under a unique United Nations Development Program (UNDP) called "TOKTEN", which stands for Transfer of Knowledge Through Expatriate Nationals. I visited India three years in a row and spent considerable amount of time in major Heart Specialty Hospitals. During my visits to India under this UNDP program, I realized that South Asians (Indians, Pakistanis, Bangladeshis, and Sri Lankans), have very high incidence of coronary artery disease [6-10]. In view of these observations, to create awareness, develop educational and prevention strategies, I started a professional society called. "South Asian Society on Atherosclerosis and Thrombosis (SASAT) in 1993, at the University of Minnesota [11]. Under the aegis of this platform, we conducted 15 international conferences in India, several half-day symposiums in other parts of the globe, in collaboration with the National Thrombosis Research Forum (Harvard University), and the International Union of Angiology (IUA), France. In this brief overview, we will discuss some salient findings of our global efforts and pose the important question, as to why we have not been able to reduce or reverse the increase in the incidence of these metabolic diseases.
The abstract of the AHA statement starts with the explanation, that South Asians make up quarter of the world's fastest growing population and are one of the fastest growing ethnic groups in the USA. They further speculate, that although they share the genetic and cultural risk factors with the South Asians abroad, South Asians living in the United States can differ in their socioeconomic status, education, health care behaviors, attitudes and health insurance, which can affect their risk and treatment and outcomes of atherosclerotic cardiovascular disease [6]. Is this a new finding by these investigators? Certainly not. Having said that, I am surprised that the authors of this AHA Statement on heart disease in South Asians, have not referred to any of our previous work on this topic for the past three decades [7-10]. This exactly is the topic, that we discussed in our very first SASAT International Conference in Mumbai in 1994. After four more such conferences in India, under the aegis of SASAT, we published our first book on the subject in India. In that book, the pioneering cardiovascular epidemiologist, Professor Henry Blackburn of the University of Minnesota, summarized our feelings in the very last sentence of his article: "The ultimate goal then is not to control disease or just reduce high risk, but to prevent high risk in the first place, among individuals and entire populations". In the same book, in my introduction, I wrote the following: Over the past decade there has been a significant decrease in illness and death from the Coronary Artery Disease (CAD) in the USA. The decline is attributed to improved health education, healthier living styles and increased physical activity. Despite this reduction in morbidity and mortality, CAD is still a major public health problem and the number one killer in many countries. Studies done in Africa, New Zealand, Singapore, Malaysia, Fiji, United Kingdom and the USA have revealed a high incidence of CAD in men and women of South Asian origin (Indians, Pakistanis, Bangladeshis and Sri Lankans) compared to other ethnic groups [7]. In view of these observations, the big question that arises in all our minds is, why is this excess of heart disease apparent in South Asians living in the USA, in spite of the better living conditions offered in the USA, compared to other countries in East Asia, Africa or Europe.

South Asians today, account for more than half of the world's cardiac patients. Heart disease is the leading cause of death in India, Pakistan, and Bangladesh, and have risen over the past several decades. South Asian immigrants to the United States develop vascular disease earlier, and it is more malignant and have higher death rates, than any other ethnic group in this country. In view of this fact, we the immigrants from South Asia, should sincerely thank American Heart Association for encouraging these experts, to put together their collective thoughts on this topic of great public health importance. In this seminal article, literature on the demographics and biological and non-biological mechanisms contributing to excess ASCVD, health behaviors, and interventions, including physical activity, diet, medication, and community strategies, in South Asians is summarized. Readers interested in this topic, are urged to go through this AHA Statement for further details [6]. The MASALA study (Mediators of Atherosclerosis in South Asians Living in 
America) is a prospective long-term ongoing study in the USA. The study found a higher incidence of Diabetes Mellitus (DM) in South Asians (23\%) compared with other ethnic groups (White 6\%, Blacks 18\%, Latinos 17\% and Chines Americans 13\%). In a separate study done as a bilateral collaborative initiative between Madras Diabetes Research Foundation (MDRF), Chennai, India, and the University of Minnesota, the authors concluded, that compared to the US, the waist-to-hip ratio is significantly higher in men and women from Chennai, India. These results support the hypothesis that Southeast Asian Indians are particularly disposed to central abdominal obesity [12].

Although number of adults with diabetes was lower in the Caucasian group, compared to other ethnic minority, number of prediabetics in the USA is not a small number. According to a recent $\mathrm{CDC}$ report, $50 \%$ of the adults in the USA are prediabetic (84 million). It is a well-known factor, that the individuals with diabetes have a several fold higher risks for developing vascular disease. In view of these observations, it is reasonable to conclude, that worldwide increase in these twin epidemics, obesity and diabetes will contribute significantly, to the increased vascular disease including CVD. According to all estimates, cardiovascular disease is the number one cause for all-cause mortality worldwide. Can we then include cardiovascular disease also as an epidemic? In 2014 there was a debate in the Legislative assembly of Manitoba, Canada about the "tsunami" of diabetes [13]. The members were concerned about the increasing healthcare cost, if the twin epidemic of obesity and diabetes are not controlled. All of the individual risk factors of CMDs pose as "tsunami" and collectively pose great challenge worldwide. I urge all readers to contemplate on this public health problem seriously, express their collective thoughts, think "out of the box", develop novel strategies, to combat these epidemics.

The purpose of writing this mini review, was not to discuss the AHA statement on the excess heart disease in immigrant South Asians living in the USA, but to elaborate on the topic of the worldwide impact of the vascular diseases and metabolic diseases. Mr. Bill Gates has an article on "Innovation for Pandemics" in the June (2018) issue of the New England Journal of Medicine, which describes the interventions developed to fight the infectious disease pandemics [14]. I wrote a comment on this article, in which I compared the economic tsunami of the metabolic disease epidemic with the pandemics that Mr. Gates referred to in his article. Every time a health risk is identified, an intervention is developed, and all out efforts are made to reduce, reverse or prevent the pandemics. How is this different when we consider the galloping increase in the incidence of metabolic disease worldwide? If we look at the seventy years of research by the Framingham heart study group, we realize that identifying modifiable risks played an important role in reducing or reversing the increases in the cardiovascular morbidity and mortality in the USA [15]. In view of these observations, guidelines and guidance statements have been developed for the management of metabolic disease risks such as hypertension, inflammation, oxidative stress, lipid abnormalities, and elevated blood glucose. Creating awareness, providing better preventive healthcare to some extent, reduced the modifiable risks such as smoking, cholesterol and blood glucose. In addition, pharma companies developed various drugs to manage modifiable risks as was the case with the pandemics. Since western medicine has failed to reduce, reverse or prevent the increase in the incidence of these diseases, using integrated approach, complimentary therapies can be introduced to provide better preventive healthcare.

In recent articles, I have argued that management of the disease is superior to the management of risk factors, a philosophy that was developed by the epidemiologists and cardiologists at the University of Minnesota. I have also stressed the importance of understanding the underlying causes of disease and disease processes, so that better and optimal (precision medicine) management strategies can be developed. Various pathophysiological cardiometabolic factors have been reported to be associated with development of acute vascular events. For instance, visceral fat is a very common feature of the South Asian phenotype. It is indeed, a metabolically active tissue that produces proinflammatory and prothrombotic cytokines. Dr. Robert Freishtat and associates at the Children's National Memorial Hospital, Washington DC, have demonstrated that visceral adipocytes shed exosomal-mediators (microRNAs) predicted to regulate key end-organ inflammatory and fibrotic pathways [16]. Inflammation has been associated with every major metabolic disease risk factor, such as subclinical atherosclerosis, oxidative stress, insulin resistance, endothelial dysfunction, hardening of the arteries, alteration of blood lipids, hypertension, and stability or the vulnerability of the vascular plaques. A comprehensive study done in 26 industrialized countries found, a significant decline in cardiovascular mortality, whereas diabetes-related mortality, contrary to all expectations, was increased in these countries [17]. According to a joint Consensus Conference Report by the American Diabetes Association (ADA), and the American College of Cardiology (ACC) Foundation, cardiometabolic risk refers to a high lifetime risk for CVDs. The specific factors that can cause this increased risk include, excess weight, obesity, insulin resistance, hyperglycemia, dyslipoproteineima, and hypertension.

According to two major professional societies, the cardiometabolic risks are no different from the modifiable risk factors for CVD, identified by the American Heart Association. In view of this commonality in risks, it is hard to understand the findings of the study we mentioned earlier in this overview, where the clinicians found a significant difference between the death due to CVDs, compared to deaths related to Diabetes [17]. Answer for this puzzle comes from a study done by the researchers of Center for Health Research, Portland, Oregon, and the researchers at Kaiser Permanente Healthcare. In this observational cohort study of 1.3 million adults, with overweight or obesity, they found four commonly observed cardiac risk factors (CRFs), the prevalence of blood pressure, elevated triglycerides, low HDL and prediabetes (32 to 60\%). An earlier ongoing study by National Health and 
Nutrition Examination Survey (NHANES) for 1999 through 2004, which included individuals with diabetes, showed that $52 \%$ of adults with overweight and $32 \%$ adults with obesity had no CRFs or only one, suggesting that various phenotypes of overweight and obesity including abdominal obesity versus subcutaneous obesity, may pose various health risks [18]. Authors of this observational cohort study concluded that, "Almost 10\% of the participants with obesity had no CRFs. Overweight or obesity increases cardiometabolic risk, but the number and type of CRFs developed varied substantially by age, even among participants with morbid obesity. In a short review like this, we will not be able to discuss all aspects of cardiometabolic syndrome, readers are urged to refer to the original articles, reviews, and meta-analysis on this subject [18-26].

Development of risk factors for cardiometabolic diseases is dynamic and unpredictable, as we have noticed from the results of various studies that we have discussed. However, one thing is certain, that is the difference between the observed mortalities between the cardiovascular, versus diabetes-related, in the 26 industrialized countries [17]. In view of the fact, that modifiable risks are addressed by the professional scientific bodies, policymakers, and healthcare providers, death due to cardiovascular disease has declined in some major countries. Whereas, death due to CVDs and diabetes-related complications has increased in low-and middle-income countries, where there no such good practices are in place. Contrary to these findings, according to the recent AHA scientific statement, South Asians living in the USA in spite of the availability of all the socioeconomic benefits, have high incidence of deaths related to CVDs and diabetes. As regards the Asian Americans living in the USA or any other countries, high prevalence of visceral obesity, insulin resistance, hypertension, type- 2 diabetes, work-related stress, and lack of physical activity or exercise, has put them at a higher risk for developing CVDs and acute vascular events. What is not clear in the AHA scientific statement is, lack of an explanation or discussion as to, why the South Asians living in the USA did not do better or as well as their US counterparts, in view of the better socio economical and living conditions in the USA? If it is the living style of these immigrants that is responsible, then the major risk factor is behavioral or work-related stress. Are there novel ways to create more effective awareness and educational programs that will address lifestyle factors in a more therapeutic manner for this high-risk ethnic group or for other ethnic groups? Do the therapies for metabolic diseases work equally well in all ethnic groups? Should we develop ethnic-specific guidelines and guidance statements for risk assessment and treatment? Just from a brief discussion on this topic regarding the excess diabetes and CVD risk in this ethnic group, it becomes evident that, contributing factors for this observed excess are not very clear and needs further exploration.

\section{Conclusion}

Comprehensive studies conducted at 26 industrialized countries, indicate a decline in the cardiovascular deaths [27].
This may be attributed to implementation of interventions aimed at modifiable risks for CVDs. The same study however, reports that in spite of this observed decline in the deaths related to CVDs, there was a significant increase in the deaths related to diabetes. Does this mean, that just management of the modifiable risks as suggested in several major studies, is not enough to reduce or reverse or prevent diabetes-related deaths? On the other hand, cardiometabolic disease risks are not very clearly defined and in view of this fact, standard care for cardiometabolic risk suffers from lack of clear, well thought out, guidelines and guidance statements. Seventy years of meticulous research by the Framingham Study Group, have defined the modifiable risks for CVDs [15]. Guidelines and guidance statements by professional societies, National Institutes of Health (NIH), and healthcare providers, have contributed significantly to the decline in deaths due to CVDs. However, deaths due to diabetes-related complications have not declined in majority of the countries. When considering the cardiometabolic risks, one should keep in mind that this is a complex cluster of disease entities, and risk assessment, risk prediction and risk management is complicated, as we do not as yet understand, all the underlying causes that promote or precipitate CRFs in these metabolic diseases. In the absence of robust guidelines specific for each of the CRFs, it is worthwhile following the success stories in CVD preventive strategies, that have worked well.

As part of the 2020 impact goals, the AHA has set out seven ideal health goals; not smoking, maintaining a normal weight, increased physical activity, a healthy diet, normal blood lipids, and a normal fasting glucose [28]. An analysis of the National Health and Nutritional Examination Survey of the USA showed that individuals who met five of the seven ideal metrics of AHA had a $78 \%$ reduction in the hazard ratio for the all-cause mortality. From the INTERHEART study under the leadership of Professor Salim Yusuf, which included 52 countries, it is estimated that modifiable risk factors account $90 \%$ of the population attributable risk for heart disease in men and $94 \%$ of the risk in women [29]. However, a recent study by Khera et al. [30] described in New England Journal of Medicine (2016) showed that in four studies with over 55,685 participants, a favorable lifestyle intervention was associated with nearly $50 \%$ lower relative risk for coronary artery disease than was with unfavorable lifestyle [30]. In conclusion, nearly $35 \%$ of the US adult population has a BMI, which indicates excess weight, and $30 \%$ of the adult population are obese worldwide, with great variation in the number and type of CRFs. We recognize an immediate need for further research to clearly define the phenotypes of excess weight, obesity (visceral obesity/ subcutaneous obesity), CRFs related to each of these phenotypes, and develop appropriate strategies for reduction, reversal and prevention of excess weight, obesity and type- 2 diabetes and its clinical complications [31-36].

\section{References}

1. Husten L (1998) Global epidemic of cardiovascular disease predicted. Lancet 352(9139): 1530. 
2. McKee M, Leon D (1999) Global epidemic of cardiovascular disease. Lancet 353(9151): 503.

3. NCD Risk Factor Collaboration (NCD-Ris-C) (2017) Worldwide trends in bod-mass index, underweight, overweight, and obesity from 1975 2016: a pooled analysis of 2416 population-based measurement studies in 128.9 million children, adolescent, and adults. Lancet 390(10113): 2627-2642.

4. NCD Risk Factor Collaboration (NCD-Ris-C) (2016) Worldwide trends in diabetes since 1980: a pooled analysis of 751 population-based studies with 4.4 million participants. Lancet 387(10027): 1513-1530.

5. Chockalingam A, Campbell NR, Fodor JG (2006) Worldwide epidemic of hypertension. Can J Cardiol 22(7): 553-555.

6. Volgman AS, Palaniappan LS, Aggarwal NT, Gupta M, Khandelwal A, et al. (2018) Atherosclerotic cardiovascular disease: in South Asians in the United States: Epidemiology, Risk factors, and treatments. A Statement from the American Heart Association. Circulation 138(1) e1-e34.

7. Rao GHR, Kakkar VV (2001) Coronary Artery Disease in South Asians: epidemiology, risk factors and prevention. Jaypee Medical Publishers, New Delhi, India.

8. Rao GHR, Thanikachalam S (2005) Coronary Artery Disease: Risk promoters, pathophysiology and prevention. Jaypee Medical Publishers, New Delhi, India.

9. Rao GHR (2016) Handbook of Coronary artery Disease. Springer Healthcare, New Delhi, India.

10. Rao GHR (2018) Coronary Artery Disease. Jaypee Medical Publishers, New Delhi, India.

11. Rao GHR (2016) Contributions of the South Asian Society on Atherosclerosis and Thrombosis and the Indian Society for Atherosclerosis Research, to our understanding of atherosclerosis and thrombosis. J Clin Prevent Cardiol 5(2): 62-72.

12. Bajaj HS, Pereira MA, Anjana RM, Deepa R, Mohan V, et al. (2014) Comparison of relative waist circumference between Asian India and US adults. J Obes 2014: 461956.

13. Gerrard JM (2016) The diabetes epidemic in Manitoba, Manitoba Liberal Caucus Report. 2014 1513-30-. Doi: 10.1016/S140-6736(16): 00618-8.

14. Gates B (2018) Innovation for Pandemics. N Engl J Med 378(22): 2057 2060.

15. Framingham Heart Study.

16. Ferrante SC, Nadler EP, Pillai DK, Hubal MJ, Wang Z, et al: (2015) Adipocyte-derived exosomal miRNAs: a novel mechanism for obesityrelated disease. Pediatrc Res 77(3): 447-454.

17. Di Cesare M, Bennet JE, Best N, Stevens GA, Danaei G, et al. (2013) The contributions of risk factor trends to cardiometabolic mortality decline in 26 industrialized countries. Int J Epidemiology 42(3): 838-848.

18. Wildman RP, Munter P, Reynolds K, McGinn AP, Rajpathak S, et al. (2008) The obese without cardiometabolic risk factor clustering and the normal weight with cardiometabolic risk factor clustering: prevalence and correlates of 2 phenotypes among US population (NHANES199-2004) Arch Int Med 168(15): 1617-1624.

19. Nichols GA, Schroeder EB, Karter AG, Gregg EW, Desai J, et al. (2015) Trends in diabetes incidence among 7 million insured adults, 20062011: the SUPREME-DM project. Am J Epidemiol 181(1): 32-39.
20. WHO Expert Consultation (2004) Appropriate body-mass index for Asian populations and its implications for policy and intervention strategies. Lancet 363(9403): 157-163.

21. Bell JA, Kivimaki M, Hamer M (2014) Metabolically healthy obesity and risk of incident type- diabetes: a meta-analysis of prospective cohort studies. Obes Rev 15(6): 504-515.

22. Despres JP (2012) What is metabolically health obesity: from epidemiology to pathophysiological insights. J Clin Endocrinol Metab 97(7): 2283-2285.

23. Lotta LA, Abbasi A, Sharp SJ, Sahlqvist AS, Waterworth D, et al. (2015) Definitions of metabolic health and risk of future type-2 diabetes in BMI categories: a systematic review and network meta-analysis. Diab Care 38(11): 2177-2187.

24. Roberson LL, Aneni EC, Maziak W, Agatston A, Feldman T, et al. (2014) Beyond BMI: the metabolically healthy obese phenotype and its association with clinical/subclinical cardiovascular disease and allcause mortality-a systematic review. BMC Publ Health 14: 14.

25. Sperling LS, Mechanick JI, Neeland IJ, Herrick CJ, Després JP, et al. (2015) The cardiometabolic health Alliance: working towards a new care model for the metabolic syndrome. J Am Coll Cardiol 66(9): 10501067.

26. Hamdy O, Porramatikul S, Al-Ozairi E (2006) Metabolic obesity: the paradox between visceral and subcutaneous fat. Curr Diab Rev 2(4): 367-373.

27. Arsenault BJ, Lachance D, Lemieux I, Alméras N, Tremblay A, et al. (2007) Visceral adipose tissue accumulation, cardiorespiratory fitness, and features of the metabolic syndrome. Arch Int Med 167(14): 15181525.

28. Lloyd Jones DM, Hong Y, Labarthe D, Mozaffarian D, Appel LJ, et al. (2010) Defining and setting national goals for cardiovascular health promotion and disease reduction: The American Heart Association's strategic impact goal through 2020 and beyond. Circulation 121(4): 586-613.

29. Yusuf S, Hawken S, Ounpuu S, Dans T, Avezum A, et al. (2004) Effect of potentially modifiable risk factors associated with myocardial infraction in 52 countries (the INTERHEART study): Case-control study. Lancet 364(9438): 937-952.

30. Khera AV, Emdin CA, Drake I, Natarajan P, Bick AG, et al. (2016) Genetic risk, adherence to a healthy lifestyle, and coronary artery disease. N Engl J Med 375(24): 2349-2358.

31. Palaniappan LP, Araneta MR, Assimes TL, Barrett-Connor EL, Carnethon MR, et al. (2010) Call to action: cardiovascular disease in Asian Americans: A science advisory from the American Heart Association. Circ 122(12): 1242-1252.

32. Rao GHR (2018) Prevention or reversal of cardiometabolic diseases. J Clin Prevent. Cardiol 7(1): 22-28.

33. Rao GHR (2018) Risk scores for acute vascular events: Expectations and Limitations. J Cardiol 2(3): 000124.

34. Rao GHR (2018) Excess weight, obesity, diabetes (type-2) and clinical complications. Arch Diab Obesity 1(1).

35. Rao GHR (2017) Type-2 diabetes, a Global epidemic. JETR. MDID555552.

36. Mohan V, Rao GHR (2007) Type-2 diabetes in South Asians: Epidemiology, Risk factors, and Prevention. JP Medical Publishers, New Delhi, India. 
CC This work is licensed under Creative

DOI: 10.19080/JOCCT.2018.12.555834
Your next submission with Juniper Publishers will reach you the below assets

- Quality Editorial service

- Swift Peer Review

- Reprints availability

- E-prints Service

- Manuscript Podcast for convenient understanding

- Global attainment for your research

- Manuscript accessibility in different formats

( Pdf, E-pub, Full Text, Audio)

- Unceasing customer service

Track the below URL for one-step submission https://juniperpublishers.com/online-submission.php 